\section{On the use of grazing-incidence small-angle X-ray scattering (GISAXS) in the morpholo- gical study of ion-implanted materials}

\author{
Francesco d'Acapito, ${ }^{\text {a* }}$ Chiara Maurizio, ${ }^{\text {a }}$ Francesco \\ Gonella, ${ }^{\text {b }}$ Elti Cattaruzza, ${ }^{\text {b }}$ Giovanni Mattei, ${ }^{\text {c }}$ Claudia \\ Mondelli, ${ }^{d}$ Alessandro Longo ${ }^{e}$ and Antonino \\ Martorana $^{\mathrm{f}}$
}

\begin{abstract}
aINFM, OGG, c/o ESRF, GILDA CRG, BP 220, F-38043 Grenoble, France, ${ }^{b}$ Università di Venezia, Dipartimento di Chimica Fisica, Dorsoduro 2137, I-30123 Venezia, Italy, 'Università di Padova, Dipartimento di Fisica, Via Marzolo 8, I-35131 Padova, Italy, ' INFM, OGG, C/o ILL, BP 156, F-38042 Grenoble, France, ${ }^{e}$ ICTPN, CNR, Via Ugo La Malfa 153, I-90146 Palermo, Italy, and ${ }^{f}$ Università di Palermo, Dipartimento di Chimica Inorganica, Viale delle Scienze, I-90128 Palermo, Italy. E-mail: dacapito@esrf.fr
\end{abstract}

Grazing-incidence small-angle X-ray scattering has become a widely used technique for the morphological analysis of surface systems. Here it is show how this technique can be applied to a buried system, like metallic clusters in glass obtained by ion implantation. The optimization of the data-collection geometry is described as well as the details of the quantitative data analysis. An experimental example on $\mathrm{Cu}+\mathrm{Au}$-implanted glasses shows the potentiality of the technique.

Keywords: grazing-incidence small-angle X-ray scattering; ion implantation; metallic nanoparticles; glass-nanocluster composites; small-angle neutron scattering.

\section{Introduction}

Composite materials consisting of nanometer-scale metallic particles embedded in glass have recently received great attention for their peculiar physical properties (Gonella \& Mazzoldi, 2000). In particular, the reduced particle size creates a number of unusual effects, like quantum confinement of the electronic wavefunctions (Alivisatos, 1996; Halperlin, 1986) or dielectric confinement (Haglund, 1995), that strongly modify the behavior of such systems with respect to the corresponding bulk forms. Applications for these materials have been proposed in optoelectronics for fast switching (Gonella \& Mazzoldi, 2000) or in magnetic recording (Haglund, 1995), to cite only a few. A key point in the construction of a nanocluster-based device is the control of the morphology in terms of cluster shape, dimension, size spread and cluster arrangement in the matrix. Laboratory techniques, like transmission electron microscopy (TEM), can provide only some of the cited parameters and in an extremely spatially reduced portion of the sample. Grazing-incidence small-angle X-ray scattering (GISAXS) is a recently developed technique (Levine et al., 1989; Naudon \& Thiaudiere, 1997) for the analysis of surface systems. Detailed descriptions of systems of particles deposited on a surface have been carried out (Robach et al., 1999; Babonneau et al., 1999, 2001; Naudon et al., 2000; Stemmer et al., 2003; Renaud et al., 2003) and a complete treatment of the GISAXS theory and analysis methods for this kind of material has been recently presented (Lazzari, 2002).

GISAXS has also found a particularly useful application in the study of ion-implanted materials, with particular attention to metal implants in glasses (d'Acapito et al., 1998; Cattaruzza et al., 2000; Maurizio, Longo et al., 2003), semiconductors in glasses and crystalline silicon (Dubcek et al., 2003; Desnica et al., 2003), and rare gases in silicon (Dubcek et al., 2004). It must be noted that, differently from surface-deposited particles, in the case of implanted materials the layer under analysis is typically a few hundreds of $\AA$ below the surface. This leads to the necessity of a non-trivial choice of the experimental conditions, in order to maximize the signal from the implanted species without being overwhelmed by that from the substrate. In this contribution we will focus on the use of GISAXS on ion-implanted materials, describing (i) the procedure to optimize the data collection, and (ii) the quantitative analysis, that takes into account the particular physical processes driving the formation of clusters in implanted substrates.

\section{Cluster formation by ion implantation}

An efficient way of obtaining nanoparticles in matrices is the ionimplantation technique (Cattaruzza, 2000). In this way, problems related to low atomic solubility or diffusion coefficients are easily overcome, practically permitting the doping of any matrix with any atomic species. In an ion-implantation process, ions with energies in the $\mathrm{keV}$ range enter the matrix and start to lose energy. In the initial part of the path, the ions transfer their kinetic energy to the matrix mainly via electronic processes. Then, when the ion has reached a sufficiently low speed, nuclear interactions dominate. Ions stop in the substrate following a distribution function, that can be parametrized by two quantities: the projected range $R_{\mathrm{p}}$ and the straggling $R_{\mathrm{s}}$. Both depend on the implantation energy $E$ and on the atomic number of the implanted species $Z$, in a way that higher $E$ leads to higher $R_{\mathrm{p}}$ and $R_{\mathrm{s}}$ values. An opposite effect on these parameters is played by $Z$. By using simulation programs based on Monte Carlo procedures it is possible to determine these values ab initio (Biersack \& Haggmark, 1980). A typical distribution of implants in a matrix is shown in Fig. 1 and was obtained using TRIM (Biersack \& Haggmark, 1980).

Another fundamental parameter in implantation processes is the fluence $\Phi$ that gives the number of ions per unit surface deposited in the material. As $R_{\mathrm{p}}$ and $R_{\mathrm{s}}$ are independent of $\Phi$ (at least for low fluences), it is also possible to calculate the volume concentration of the implant given a suitable set of initial conditions. The formation of

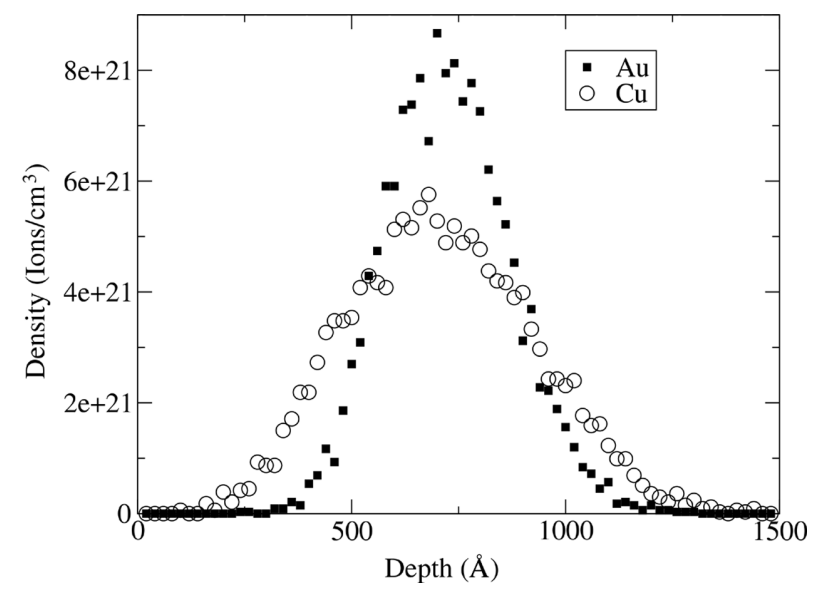

Figure 1

Simulation of the implant profile of a $\mathrm{SiO}_{2}$ glass implanted with $100 \mathrm{keV}$ $\mathrm{Cu}^{+}$ions at a fluence of $3 \times 10^{16}$ atoms cm$~_{-2}$ and $210 \mathrm{keV} \mathrm{Au}^{+}$ions at the same fluence. The simulation was made using the TRIM program (Biersack \& Haggmark, 1980). The projected range $R_{\mathrm{p}}$ and straggling $R_{\mathrm{s}}$ are $R_{\mathrm{p}}=700 \AA$ and $R_{\mathrm{s}}=216 \AA$ for $\mathrm{Cu}$ and $R_{\mathrm{p}}=720 \AA$ and $R_{\mathrm{s}}=144 \AA$ for $\mathrm{Au}$. 
clusters in the matrix can be seen, in a simplified way, as a diffusion process followed by a chemical interaction. The implanted atomic species, after deposition, start to migrate in the matrix at a rate determined by their diffusion constant $D_{\text {c }}$. When two diffusing atoms come in contact and the metal Gibbs free energy is lower than that of the related oxides (if implanting an oxide matrix), they start to form a nucleation center for the future metallic cluster. This process, which already exists during the implantation, can be continued with a subsequent annealing of the material. The first step, of kinematical nature, can be described by stating that the average distance $l$ between two atoms (that depend on their volume density $\rho$ ) must be smaller than the diffusion length $\ell$,

$$
\ell=\left(D_{\mathrm{c}} t\right) \gg(3 \pi / 4 \rho)^{1 / 3}=l .
$$

$\ell$ is determined by the diffusion coefficient $D_{\mathrm{c}}$ and by the treatment time $t$, as stated in (1).

The second condition means that the compound we wish to form in the matrix (metallic cluster, namely) is thermodynamically more stable than other possible (oxides) compounds, i.e.

$$
\Delta G_{\text {metal }}<\min \left(\Delta G_{\text {oxides }}\right) .
$$

This picture represents an idealized model for the matrix-implant interaction and accounts for the simplest processes. The following more complex phenomena are not accounted for:

(i) The matrix damage owing to the implant can locally alter the distribution of chemical species leading to a modified behavior of the implant from what is foreseen for the unperturbed matrix. An example of this effect is described by d'Acapito et al. (2000).

(ii) The surface atoms are usually a considerable fraction of the total atoms in the cluster, so phenomena like formation of core-shell clusters or de-alloying of mixed component particles are likely to be formed during particular treatments (Mattei et al. 2003).

\section{The GISAXS technique applied to implanted materials}

The method is based on the observation that X-rays have a refraction index $n$ of less than 1 (Parratt, 1954),

$$
n=1-\delta-\iota \beta,
$$

where $\delta$ and $\beta$ are the so-called anomalous dispersion corrections. The fact that the real part of $n$ is smaller than 1 can be seen, in a semiclassical way, as originating in the lag in the response of the bound electrons to the electric field that oscillates at a frequency higher than their typical resonance value (Feynman et al., 1970). The common laws for refraction and reflection still hold, but the material plays the role of the low $n$ zone and the vacuum that of the high $n$ zone. In particular, the total external reflection of a beam impinging with an angle $\varphi$ lower than a critical value $\varphi_{\mathrm{c}}$ is possible. In this condition the components of the wavevector $\mathbf{k}$ of the refracted beam parallel to the surface $\left(k_{x}, k_{y}\right)$ are real, whereas the component perpendicular to the surface $\left(k_{z}\right)$ is imaginary. Thus, the beam propagates parallel to the surface and penetrates in the material with an exponentially damped wave described by a damping parameter $z_{1 / e}$. By increasing the incidence angle, $k_{z}$ starts to develop a non-zero real part and propagation sets up also inside the material (Parratt, 1954).

As already pointed out, when dealing with implanted materials the interesting part is only a thin layer embedded in a much thicker (about four orders of magnitude) substrate. The problem is then to confine the probe beam into a zone slightly below the surface, where the atomic density of the implanted species is maximum, minimizing the contribution of the substrate. Indeed, when working in normal incidence the substrate signal overwhelms the signal from the implanted layer, whereas, when working in total reflection conditions, the limited penetration prevents the probe beam from joining the implanted region. Examples of these phenomena have already been shown by d'Acapito \& Zontone (1999) in the case of X-ray diffraction. For this reason, when dealing with ion-implanted materials, the incidence angle has to be optimized to correctly carry out this kind of experiment. This is achieved by analyzing a typical reflectivity curve, shown in Fig. 2, relative to a heavy ion implant in a light matrix. In the low-angle region the reflectivity is maximum as this is the total reflection regime for the glass/air interface. Above $0.2^{\circ}$ the signal starts to decrease, indicating that the beam is penetrating the glass. However, it is still reflected at the glass/implanted-region interface, as evidenced by the presence of Kiessig fringes (Kiessig, 1931) originated by the interference between the beam partially reflected at the air/glass interface and that reflected at the glass/implanted-region interface. The observation of the fringes in this kind of sample would seem surprising, since the glass/implanted-region interface is not sharp but has a roughly Gaussian shape, as shown in Fig. 1. However, it must be noted that, owing to the low scattering angle, the value of the exchanged wavevector $q$ is also low, of the order of $\sim 0.03 \AA^{-1}$ in the present case, corresponding to a spatial resolution of $\sim 210 \AA$. This value is well comparable with the straggling of the implant profile, so the probe beam sees the system as an almost sharp interface. Clearly, owing to the diffuseness of the interface, the intensity of the Kiessig fringes is much lower than that observed in abrupt interfaces. The presence of the maxima indicates that, in the related angular zone, the beam is confined to the implanted region and can be used for structural studies with enhanced spatial sensitivity to the region of interest. Confirmation of this can be found in Fig. 3, which represents the intensity (in the substrate) of the refracted electric field in a two-dimensional incidence-angle-versus-depth space. Here, a high field intensity zone can be seen at a depth corresponding to the implanted region when the incidence angle is at a value near to the first fringe. Thus, by choosing the incidence angle on this point, a maximum sensitivity to the implanted region is obtained permitting experiments of X-ray diffraction or scattering. d'Acapito \& Zontone (1999) report the effect of the choice of the incidence angle on the diffraction spectrum from an Ag-implanted sample, showing the maximization of the signal when working on the first fringe.

\section{Data collection and analysis}

A typical experimental setup for the collection of GISAXS data consists of a precise sample mounting stage that permits the alignment of the sample in total reflection condition and a detector for specular reflectivity. The sample has to be aligned in a suitable way, as shown in the previous section, in order to maximize the sensitivity to the implanted region. After the collection of a reflectivity curve, and the choice of the correct incidence angle, the small-angle scattering spectrum can be collected. Both linear or two-dimensional detectors are suitable for this purpose even if, using the latter kind, a more efficient use of beam time is obtained, since the entire scattering pattern is recorded in a single shot. Typical integration times on an undulator beamline from a third-generation source (here, the European Synchrotron Radiation Facility, ESRF) are of the order of 10 min. Typical GISAXS spectra from ion-implanted glasses are shown in Figs. 4 and 5.

This kind of data permits the extraction of detailed information on the morphology of the particles, as already pointed out in the case of surface-deposited particles (Robach et al., 1999) or oriented ellipsoids in glass (Polizzi et al., 1998). When dealing with ion-implanted 
systems, different spatial distributions of the particles in the substrate can be observed. For example, the ring visible in the scattering pattern of Fig. 4 illustrates the presence of a well defined interparticle spatial correlation length; the radial symmetry indicates a uniform distribution of particles in space. When the particles are arranged in a layer, only in-plane correlations are visible and the scattering pattern takes the form shown in Fig. 5. In most cases, however, ion implantation in glassy systems leads to the formation of spherical particles distributed in a three-dimensional way. A consequence of this is that the obtained scattering patterns have a radial symmetry, and this can be exploited in the data analysis. The first step consists of reducing the two-dimensional data to a conventional one-dimensional scattering curve. For this reason, data have to be integrated at constant exchanged wavevector $q$ over the various spatial orientations. The origin of the $q$ scale can be determined by finding the center of the particle correlation ring using routines available on image-analysis programs (Hammersley et al., 1996), or by trials by angularly inte-

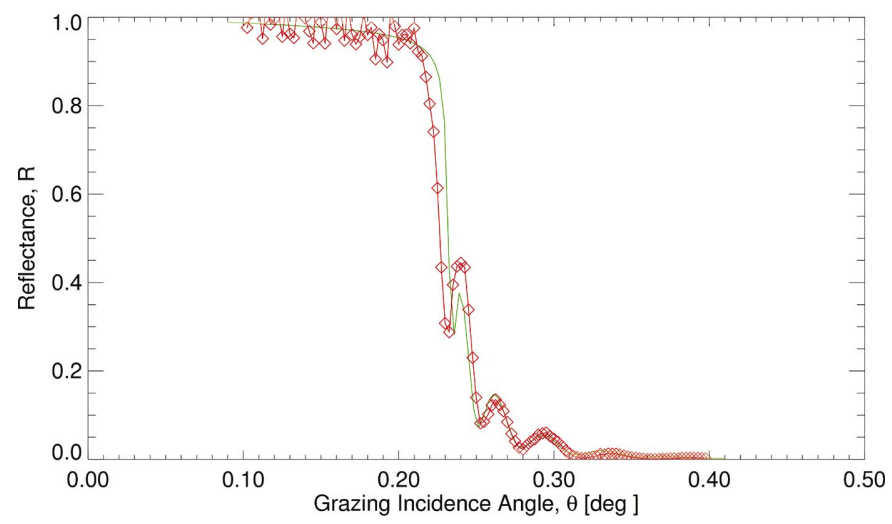

\section{Figure 2}

Specular reflectivity spectrum recorded at $8325 \mathrm{eV}$ on a $\mathrm{SiO}_{2}$ glass doubly implanted with $100 \mathrm{keV} \mathrm{Cu}{ }^{+}$and $100 \mathrm{keV} \mathrm{Ni}^{+}$ions each at a fluence of $2 \times 10^{16}$ ions $\mathrm{cm}^{-2}$. The superimposed curve is a fit to the experimental data made using the IMD program (Windt, 1998). The system was modeled by a glass layer of thickness $w_{g}=694 \AA$ and an implanted region of thickness $w_{\mathrm{i}}=$ $114 \AA$ of composition $\mathrm{SiO}_{2} \mathrm{Cu}_{0.05} \mathrm{Ni}_{0.05}$. All these data, together with the roughness of the layers $\sigma$, were refined to reproduce the reflectivity curve. The model sample obtained in this way was then used for subsequent calculations.
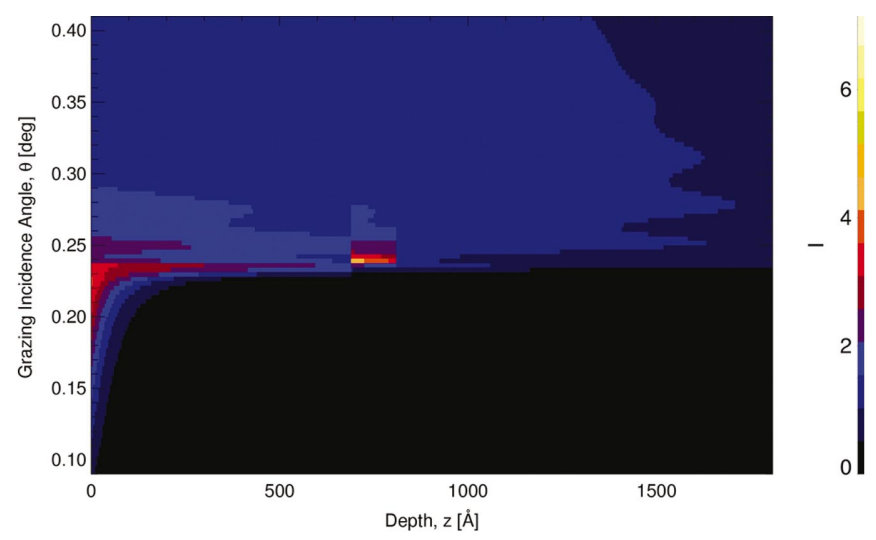

\section{Figure 3}

Simulation of the electric field intensity in the implanted glass shown in Fig. 2 at various incidence angles. The intensity (color) is plotted against incidence angle and depth. The model for the glass was derived from the fit of the reflectivity curve shown in Fig. 2. Note that the maximum intensity in the implanted layer appears for incidence angles around $0.24^{\circ}$ corresponding to the first Kiessig fringe. grating two sectors opposite to the symmetry axis to obtain two curves with coinciding maxima. At this point we have to consider how refraction processes enter into the data-collection procedure. First of all, the direction of the probe beam (refracted in the substrate) is slightly different from the direction of the direct beam. Moreover, the outgoing (scattered) beams undergo a refraction at the glass-air interface, mainly at low scattering angles. The result of these two facts is that the $q$ scale, in the so-calculated monodimensional scattering curve, needs to be suitably corrected in order to obtain reliable quantitative results. The problem of GISAXS data corrections related to refraction processes has been presented in the literature

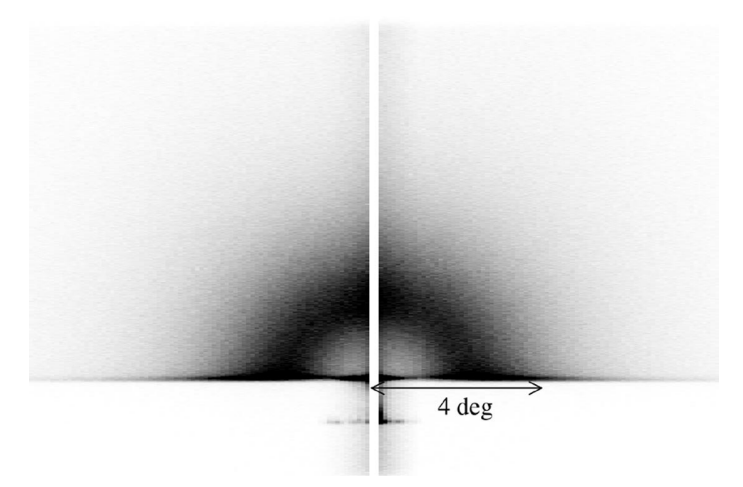

Figure 4

GISAXS pattern from an $\mathrm{Au}+\mathrm{Cu}$-implanted silica (implantation fluence $=$ $3 \times 10^{16}$ ions $\mathrm{cm}^{-2}$ for each ion species, implantation energy $=90 \mathrm{keV}$ and $190 \mathrm{keV}$ for $\mathrm{Cu}^{+}$and $\mathrm{Au}^{+}$ions, respectively). The data were collected on the ID10B beamline at ESRF, using an $8.0 \mathrm{keV}$ X-ray beam and scanning horizontally a position-sensitive linear detector $\left(0.05^{\circ}\right.$ step $)$ to collect the twodimensional pattern. Here a uniform ring is evident, indicating a threedimensional distribution of particles. The angular scale, indicated below the scattering ring, is identical for the $x$ and $y$ directions and $1^{\circ}$ corresponds to $0.07 \AA^{-1}$.
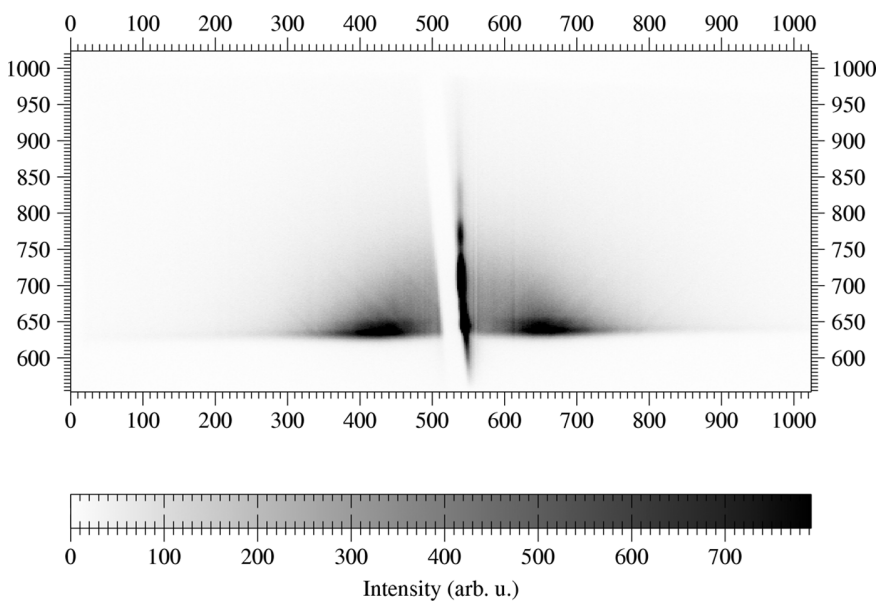

Figure 5

GISAXS scattering pattern on a $\mathrm{Cu}+\mathrm{Co}$-implanted silica (implantation fluence $=6 \times 10^{16}$ ions $\mathrm{cm}^{-2}$ for each ion species, implantation energy $=$ $100 \mathrm{keV}$ and $90 \mathrm{keV}$ for $\mathrm{Cu}^{+}$and $\mathrm{Co}^{+}$ions, respectively). The data were collected on the ID1 beamline at ESRF using an $8.0 \mathrm{keV}$ beam and a twodimensional gas-filled detector. The sample-to-detector distance was $3250 \mathrm{~mm}$, the coordinates indicate the pixel number $(1$ pixel $=200 \mu \mathrm{m}$, 100 pixels $=0.025 \AA^{-1}$ ) and the gray scale is linear with the scattered intensity. In this case the scattering pattern consists only of a pair of side lobes, indicating that the particles are arranged in a plane parallel to the glass surface. 
and procedures have been published by Kutsch et al. (1997) and Martorana et al. (2001).

A typical example of a monodimensional scattering curve is shown in Fig. 6. The ensemble of particles, created in a substrate by ion implantation, has peculiar features that must be kept in mind for a correct quantitative analysis:

(i) The particles have a spherical shape and grow by a diffusion process. They reasonably form an ensemble of hard non-penetrating spheres.

(ii) The average particle-particle correlation distance is of the same order of magnitude as the particle size. Both the particle structure factor $S$ and form factor $\Phi_{f}$ contribute in the same $q$ region.

(iii) The particle distribution size is quite wide, its width being a considerable fraction of the mean value. From TEM images a correlation between particle size and depth in the substrate is evident, its details depending on the preparation parameters (Fig. 7).

A suitable model for data analysis has been developed, taking into account the previous points. The model proposed by our group, see the analysis of $\mathrm{Cu}+\mathrm{Ni}$-implanted samples by Cattaruzza et al. (2000), describes the scattered intensity $I(q)$ as follows,

$$
I(q)=\Delta \rho^{2} \int_{0}^{\infty} \Phi_{f}(q, R)^{2} S\left[q, R_{\mathrm{HS}}(R), \eta_{\mathrm{HS}}\right] N(R) \mathrm{dR},
$$

where $\Phi_{f}(q, R)$ is the particle form factor, $S\left[q, R_{\mathrm{HS}}(R), \eta_{\mathrm{HS}}\right]$ is the particle ensemble structure factor and $N(R)$ is the particle size distribution. For each size $R$, we calculate a scattering curve $I(R)$ as the product between $\Phi_{f}$ and $S$ and then we integrate over the size distribution. This corresponds to the so-called 'local monodisperse approximation' (LMA) (Pedersen, 1993, 1994), which provides a better agreement with experimental data than the 'decoupled approximation' (DA) (Cattaruzza et al., 2000). The observation

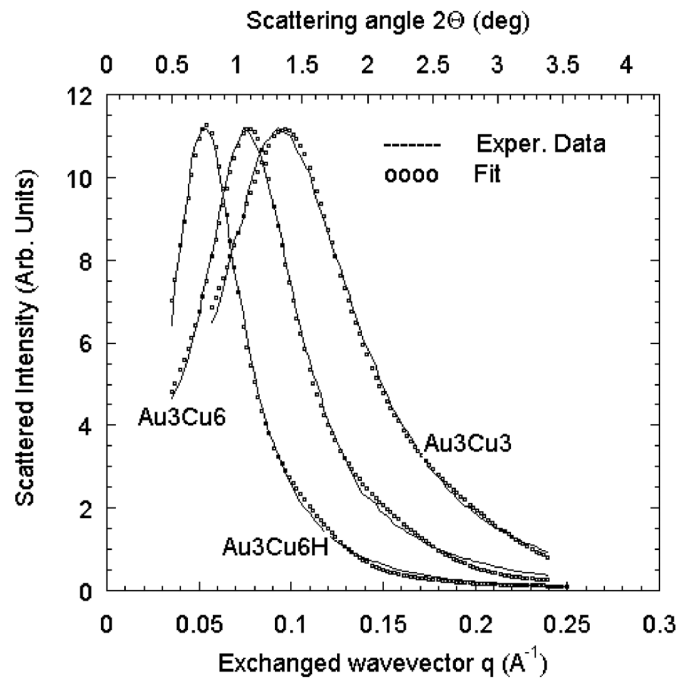

\section{Figure 6}

Small-angle X-ray scattering profiles (line) recorded in grazing-incidence mode on a set of $\mathrm{Au}+\mathrm{Cu}$-implanted silica slides. In the sample name the number after each element symbol indicates the corresponding implantation dose in units of $10^{16}$ ions $\mathrm{cm}^{-2}$. The sample Au3Cu6H was heated in $\mathrm{H}_{2}(4 \%)$ atmosphere for $1 \mathrm{~h}$ at $1173 \mathrm{~K}$ after sequential $\mathrm{Au}+\mathrm{Cu}$ implantation. The twodimensional spectra were recorded on the ID10B beamline (ESRF), then radially integrated to obtain monodimensional profiles, accounting for the $\mathrm{X}$-ray refraction at sample surface (Martorana et al., 2001). The curves are scaled to a common amplitude of the maximum for an easier comparison. The results of the best fits with the LMA model, and whose parameters are shown in Table 1, are also shown (circles). recalled in (iii) justifies the use of this model. Looking in more detail, we use the sphere form factor (Guinier \& Fournet, 1955)

$$
\Phi_{f}(q, R)=4 \pi[\sin (Q R)-Q R \cos (Q R)] / Q^{3},
$$

whereas the particle ensemble $S\left(q, R_{\mathrm{HS}}, \eta_{\mathrm{HS}}\right)$ is described by the well known Percus-Yevick structure factor (Percus \& Yevick, 1958; Ashcroft \& Lekner, 1966; Pedersen, 1993; Maurizio, Longo et al., 2003). Here, $\eta_{\mathrm{HS}}$ is the hard sphere (HS) packing fraction (particles per unit volume) and $R_{\mathrm{HS}}$ is their radius. It should be noted that $R_{\mathrm{HS}}$ is different from the particle radius $R$, and, in particular, is greater. This is because, as the particles grow with a diffusion process, i.e. the metal ions leave the glass matrix to migrate in the particle, a glass corona strongly depleted in metal ions tends to form around bigger particles. So, in an ideal fully aggregated system, all the particles are separated by spaces of pure substrate. The ratio between the particle radius and the hard-sphere radii (particle + depletion corona) can be calculated by supposing that all the ions originally contained in the hard-sphere volume (determined by the local density $\rho_{\mathrm{i}}$ achieved at the end of the implant process) collapse in the central cluster. This cluster exhibits the typical metal density $\rho_{\mathrm{m}}$ and from this we derive

$$
R_{\mathrm{HS}} / R=\left(\rho_{\mathrm{m}} / \rho_{\mathrm{i}}\right)^{1 / 3} .
$$

This ratio can be determined a priori by the density estimated by simulations of the implant and varied only slightly during the fitting process. Eventually the scattering curves calculated in this way are averaged over the particle size distribution, which in our case is described by a Weibull function (Pedersen, 1993) as shown here,

$$
N(R)=\left(R / R_{\mathrm{m}}\right)^{b-1} \exp \left[-\left(R / R_{\mathrm{m}}\right)^{b}\right],
$$

where $R_{\mathrm{m}}$ is the average value of the particle size and $b$ is a parameter that controls the distribution width and shape (Pedersen, 1993). All these parameters have different effects on the calculated scattering curve. Different values of $R_{\mathrm{m}}$ affect the slope of the $\ln [I(q)]$ versus $q^{2}$ curve at low exchanged momentum data above the correlation peak (bigger values of $R_{\mathrm{m}}$ lead to steeper curves), whereas the value of $\eta_{\mathrm{HS}}$ affects the position of the correlation peak (for higher packing fractions the peak shifts at higher $q$ values). The $b$ parameter affects the width of the particle size distribution function: for higher values a narrower distribution is obtained around $R_{\mathrm{m}}$, and shape oscillations start to show up in the high- $q$ part of the scattering curve. Correlations among the parameters are easily checked and accounted for in the error estimation, by using suitable codes [like MINUIT (1994)]

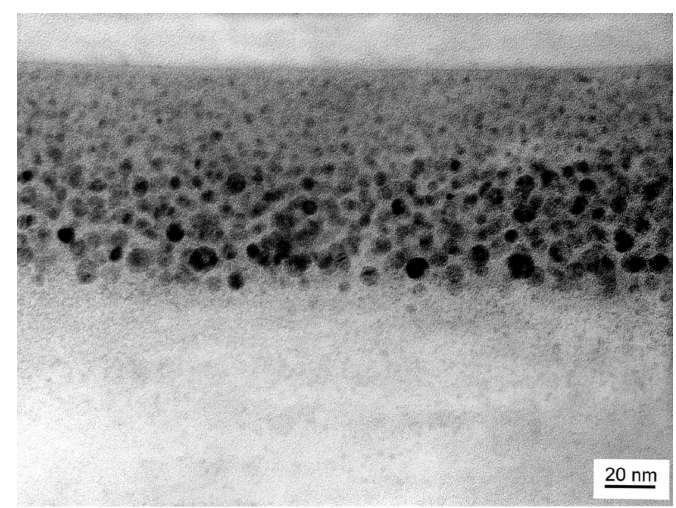

Figure 7

Example of the typical morphology of a metal-implanted glass. Here a crosssectional bright-field TEM micrograph of a $\mathrm{Cu}+\mathrm{Ni}$-implanted silica sample is shown. The implantation energies were $90 \mathrm{keV}$ and $100 \mathrm{keV}$ for $\mathrm{Cu}$ and $\mathrm{Ni}$ ions, respectively. The implantation fluences were $6 \times 10^{16}$ ions cm $\mathrm{cm}^{-2}$ for each ion species. 
for the curve fitting. In the data-fitting process, a further scale factor $S$ and a constant background $B$ are added to the modelling function. In principle it would be possible to use absolute intensities as is usual in measurements collected in transmission mode. However, a number of poorly controlled parameters contribute to prevent a reliable determination of absolute intensities when working at the critical angle:

(i) The scattered intensity depends on the intensity of the probe beam. This, in turn, strongly depends on the incidence angle at values near the critical value and on the details of the electronic density of the material. Both quantities are known only at an approximate level in most cases.

(ii) The surface roughness can contribute to modify the intensity of the refracted beam as well as to add a background to the scattering pattern.

For this reason we preferred to perform the data analysis without considering absolute intensities. It is worth noticing, at this point, that no scattering cross section of the particle system and glass are cited in the whole treatment, making it suitable to both neutron and X-ray scattering. The experiment in the former case is strongly dependent on the achievement of a sufficient contrast between the particles and the matrix, a condition that can be actually satisfied in some cases as we will show in the next section. The model discussed can be used to process GISAXS (and SAXS) data to obtain some structural parameters from the investigated samples, such as the cluster size distribution and the intercluster mean distance. In principle this technique presents some advantages with respect to common TEM analysis, because it is not destructive, it averages over a macroscopic number of particles and permits a direct determination of the interparticle correlation distance. Moreover, time-resolved experiments (morphological evolution under thermal annealing or similar) can be carried out considering the relatively short acquisition time (Renaud et al., 2003).

\section{Experimental examples}

Here we show an example of how GISAXS can be used in the investigation of ion-implanted glasses. Silica substrates were implanted with $\mathrm{Cu}$ and $\mathrm{Au}$ ions at energies of $90 \mathrm{keV}$ and $190 \mathrm{keV}$, respectively, and at different fluences $\Phi$. These implantation parameters determine a good overlap of the two implantation profiles in the sample. Three samples are described: the first (Au3Cu3) implanted with the same amount of $\mathrm{Cu}$ and $\mathrm{Au}(\Phi=3 \times$ $10^{16}$ ions $\mathrm{cm}^{-2}$, the second (Au3Cu6) rich in $\mathrm{Cu}\left(6 \times 10^{16} \mathrm{Cu}^{+} \mathrm{cm}^{-2}+\right.$ $\left.3 \times 10^{16} \mathrm{Au}^{+} \mathrm{cm}^{-2}\right)$ and the third $(\mathrm{Au} 3 \mathrm{Cu} 6 \mathrm{H})$ identical to the second and annealed, after implantation, in a reducing atmosphere $\left[\operatorname{Ar}_{(0.96)} \mathrm{H}_{2(0.04)}\right.$ at $1173 \mathrm{~K}$ for $\left.1 \mathrm{~h}\right]$.

The fitting to the data was carried out in the framework of the LMA model, as described in the previous section, by using as physical-free parameters the hard-sphere packing fraction $\eta_{\mathrm{HS}}$, the radius of the metallic sphere $R_{\mathrm{m}}$, the ratio between the hard-sphere radius $R_{\mathrm{HS}}$ and $R_{\mathrm{m}}, k_{\mathrm{c}}$, and the Weibull size distribution parameter $b$. Added to these, two non-physical free parameters, namely a background and a scaling factor, were also used. An initial guess for $k_{\mathrm{c}}$ was derived by knowing that the ratio between the densities of the metals in the glass and bulk form was roughly $6: 1\left(k_{\mathrm{c}}=1.8\right)$. The code uses the MINUIT CERN routine $($ MINUIT, 1994) to minimize the sum of the squares of the differences between the experimental data and the model; linear or logarithmic scale fits can be chosen. Errors were derived from a $\chi^{2}$ function analysis at a $68 \%$ confidence level. The results of the analysis are shown in Table 1 . Here, $\eta_{\mathrm{HS}}, k_{\mathrm{c}}, R_{\mathrm{m}}$ and $b$ are the actual fit parameters, the others (the hard-sphere radius $R_{\mathrm{HS}}$, the metallic-sphere packing fraction $\eta$, the metallic-sphere interparticle distance $D$ ) are derived from these values using the following equations,

$$
\begin{aligned}
R_{\mathrm{HS}} & =k_{\mathrm{c}} R_{\mathrm{m}}, \\
\eta & =\eta_{\mathrm{HS}} / k_{\mathrm{c}}^{3}, \\
D & =2 R_{\mathrm{HS}} / \eta_{\mathrm{HS}}^{1 / 3} .
\end{aligned}
$$

We recall that the $\eta$ values for the FCC, BCC, diamond and gas at normal conditions are $0.74,0.68,0.34$ and 0.0007 , respectively. The sequential $\mathrm{Au}+\mathrm{Cu}$ ion implantation in silica (samples $\mathrm{Au} 3 \mathrm{Cu} 3$, $\mathrm{Au} 3 \mathrm{Cu} 6 \mathrm{H}$ ) determines the formation of $\mathrm{Au}-\mathrm{Cu}$ alloy nanoclusters (Gonella et al., 1999). The increase of the fluence of the second implanted ion leads to the formation of larger and more separated alloy clusters (see Table 1). There are different facts that explain this result. First, the high-fluence implantation determines an increase in the $\mathrm{Cu}$ concentration in the implanted region, thus promoting the dopant aggregation; and second, the irradiation process itself enhances the diffusion of the $\mathrm{Cu}$ atoms into the alloy clusters. In the $\mathrm{Au} 3 \mathrm{Cu} 3$ sample the alloy clusters are poor in $\mathrm{Cu}$ because a significant fraction of $\mathrm{Cu}$ atoms are dispersed and oxidized in the matrix (Gonella et al., 1999; Maurizio, Mattei et al., 2003), whereas in the case of higher $\mathrm{Cu}$ implantation (sample Au3Cu6) the alloy cluster average composition is rich in $\mathrm{Cu}$ (Maurizio, Mattei et al., 2003), indicating that the fraction of $\mathrm{Cu}$ atoms that participate in the alloy is higher than in the first case. The effect of the annealing in $\mathrm{H}_{2}(4 \%)$ atmosphere is to promote the cluster growth; moreover, the clusters after $1 \mathrm{~h}$ annealing (sample Au3Cu6) are less packed than in the asimplanted sample (sample Au3Cu6H, see Table 1). This behavior is what is expected from a diffusive growth where larger units grow at the expense of the smaller ones. In this case the annealing induces both a chemical reduction of the $\mathrm{Cu}$ atoms dispersed in the matrix and a diffusion and aggregation of the dopant. As a final point, it is worth noting that differences are generally evidenced between the mean cluster size obtained by TEM and GISAXS analyses. These are due not only to the fact that the two techniques investigate different population of clusters, but also to the fact that in the expression (4) of the scattered intensity the number of particles $N(R)$ with size $R$ is weighted with the sixth power of the cluster radius, so that the contribution of the larger particles to the scattering pattern is dominant. For a detailed comparison of GISAXS and TEM results for ion-implanted silica, the reader is referred to Maurizio, Longo et al. (2003).

\section{Application to neutron scattering}

In the formulation of the model presented above, no explicit reference to scattering factors was made owing to the fact that, with this technique, the absolute scattering intensities are difficult to measure. Thus the model uses an arbitrary scale factor to reproduce the data. This also means that data coming from a neutron-scattering experi- 


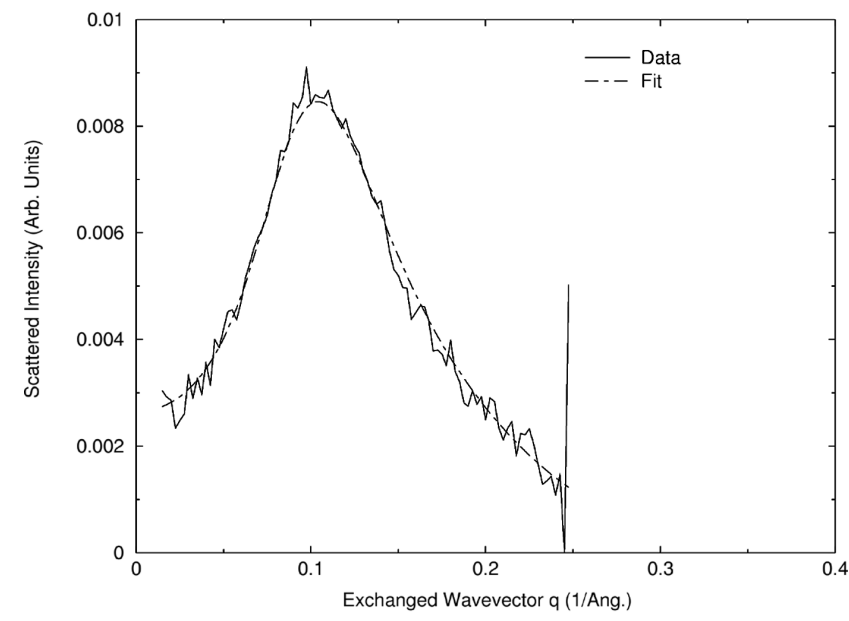

Figure 8

Neutron-scattering data from Ni-implanted silica sample. The best-fit curve is also shown.

ment could in principle be treated in the same way. A test experiment was carried out in transmission mode, on a $6 \times 10^{16} \mathrm{Ni} \mathrm{cm}^{-2}$ implanted silica glass to check the feasibility of the technique. The $\mathrm{Ni}$ implant was chosen because of the relatively large scattering length of $\mathrm{Ni}\left(18.5\right.$ barns atom $\left.^{-1}\right)$ compared with those of $\mathrm{Si}$ $\left(2.617\right.$ barns atom $\left.^{-1}\right)$ and $\mathrm{O}$ (4.232 barns atom $\left.{ }^{-1}\right)$. Even if the restrictions on the scattering lengths represent a serious limitation to the applicability of this technique, the possibility of performing spindependent scattering opens new scenarios in the study of magnetic clusters (de Julian et al., 2001; Maurizio, Longo et al., 2003). The experiment was carried out at the D22 beamline at the Institut Laue Langevin (ILL). The neutron wavelength was $\lambda=8 \AA$ and the detector was placed $3 \mathrm{~m}$ from the sample. The scattering data were quantitatively analyzed with the LMA model and the fit quality is shown in Fig. 8. We obtained a metallic-sphere radius $R_{\mathrm{m}}=11 \AA$, an interparticle distance $D=75 \AA$ and a packing fraction $\eta=0.024$.

\section{Conclusion}

In this paper we have presented an introduction to the use of the grazing-incidence small-angle X-ray scattering technique in the morphological study of metal-glass composites obtained by ion implantation. The buried nature of these samples requires a particular optimization of the data-acquisition system in order to collect meaningful data. By suitably choosing the incident angle, integrating the two-dimensional detector data and applying the refraction corrections, scattering functions can be obtained from which quantitative morphological parameters can be extracted. The model for data analysis is based on a Percus-Yevick distribution of polydispersed hard spheres. The size distribution is of the Weibull type and the local monodispersed approximation is used. In the test case of $\mathrm{Cu}+\mathrm{Au}$-implanted glasses, parameters like the metallic particle radius, the interparticle distance and packing fraction have been determined. We have shown that the model is also suitable for the analysis of neutron-scattering data, which will open the possibility of performing studies on magnetic structures on magnetic clusters.

The authors are indebted to the staff of the ID10 and ID01 beamlines at the ESRF (D. Thiaudiere, D. Smilgies, O. Konovalov, F. Zontone, P. Boesecke and T. H. Metzger) and to R. May of the D22 beamline at ILL for skillful support and fruitful discussions.

\section{References}

d'Acapito, F., Mobilio, S., Battaglin, G., Cattaruzza, E., Gonella, F., Caccavale, F., Mazzoldi, P. \& Regnard, J. R. (2000). J. Appl. Phys. 87, 1819-1824.

d'Acapito, F., Thiaudiere, D., Zontone, F. \& Regnard, J. R. (1998). Mater. Sci. Forum, 278/281, 891-896.

d'Acapito, F. \& Zontone, F. (1999). J. Appl. Cryst. 32, 234-240.

Alivisatos, P. (1996). Science, 271, 933-937.

Ashcroft, N. W. \& Lekner, J. (1966). Phys. Rev. 145, 83-90.

Babonneau, D., Naudon, A., Thiaudiere, D. \& Lequien, S. (1999). J. Appl. Cryst. 32, 226-233.

Babonneau, D., Videnovic, I. R., Garnier, M. \& Oelhafen, P. (2001). Phys. Rev. $B, \mathbf{6 3}, 195401-195405$.

Biersack, J. P. \& Haggmark, L. G. (1980). Nucl. Instrum. Methods, 174, $257-$ 269.

Cattaruzza, E. (2000). Nucl. Instrum. Methods B, 169, 141-155.

Cattaruzza, E., d'Acapito, F., Gonella, F., Longo, A., Martorana, A., Mattei, G., Maurizio, C. \& Thiaudiere, D. (2000). J. Appl. Cryst. 33, 740-743.

Desnica, U. V., Dubcek, P., Desnica-Frankovic, I. D., Buljan, M., Salamon, K., Milat, O.., Bernstoff, S. \& White, C. W. (2003). Nucl. Instrum. Methods B, 200, 191-195.

Dubcek, P., Desnica, U. V., Desnica-Frankovic, I. D., Bernstoff, S. \& Meldrum, A. (2003). Nucl. Instrum. Methods B, 200, 138-141.

Dubcek, P., Pivac, B., Milat, O., Bernstoff, S. \& Zulim, I. (2004). Nucl. Instrum. Methods B, 215, 122-128.

Feynman, R. P., Leighton, R. B. \& Sands, M. (1970). The Feynman Lectures on Physics. New York: Addison-Wesley.

Gonella, F., Mattei, G., Mazzoldi, P., Sada, C., Battaglin, G. \& Cattaruzza, E. (1999). Appl. Phys. Lett. 75, 55-57.

Gonella, F. \& Mazzoldi, P. (2000). Handbook of Nanostructured Materials and Nanotechnology, Vol. 4. San Diego: Academic Press.

Guinier, A. \& Fournet, G. (1955). Small Angle Scattering of X-rays. New York: Wiley.

Haglund, R. (1995). Handbook of Optical Properties, Vol. II. Boca Raton: CRC Press.

Halperlin, W. (1986). Rev. Mod. Phys. 58, 533-606.

Hammersley, A. P., Svensson, S., Hanfland, M., Fitch, A. \& Hausermann, D. (1996). High Press. Res. 14, 235-248.

Julian, C. de, Sangregorio, C., Mattei, G., Battaglin, G., Cattaruzza, E., Gonella, F., Russo, S. L., D’Orazio, F., Lucari, F., De, G., Gatteschi, D. \& Mazzoldi, P. (2001). J. Magn. Magn. Mater. 226/230, 1912.

Kiessig, H. (1931). Ann. Phys. 10, 769-788.

Kutsch, B., Lyon, O., Schmitt, M., Mennig, M. \& Schmidt, H. (1997). J. Appl. Cryst. 30, 948-956.

Lazzari, R. (2002). J. Appl. Cryst. 35, 406-421.

Levine, J. R., Cohen, J. B., Chung, Y. W. \& Georgopoulos, P. (1989). J. Appl. Cryst. 22, 528-532.

Martorana, A., Longo, A., d'Acapito, F., Maurizio, C., Cattaruzza, E. \& Gonella, F. (2001). J. Appl. Cryst. 34, 152-156.

Mattei, G., Marchi, G. D., Maurizio, C., Mazzoldi, P., Sada, C., Bello, V. \& Battaglin, G. (2003). Phys. Rev. Lett. 90, 85502-85505.

Maurizio, C., Longo, A., Martorana, A., Cattaruzza, E., d'Acapito, F., Gonella, F., de Julian, C., Mattei, G., Mazzoldi, P., Padovani, S. \& Boesecke, P. (2003). J. Appl. Cryst. 36, 732-735.

Maurizio, C., Mattei, G., Mazzoldi, P., Padovani, S., Cattaruzza, E., Gonella, F., d'Acapito, F. \& Zontone, F. (2003). Nucl. Instrum. Methods B, 200, 178-184

MINUIT (1994). CERN Program Library Long Writeup D506. CERN, Geneva, Switzerland.

Naudon, A., Babonneau, D., Thiaudiere, D. \& Luien, S. (2000). Physica B, 283, 69-74.

Naudon, A. \& Thiaudiere, D. (1997). J. Appl. Cryst. 30, 822-827.

Parratt, L. (1954). Phys. Rev. 95, 359-369.

Pedersen, J. (1993). Phys. Rev. B, 47, 657-665.

Pedersen, J. (1994). J. Appl. Cryst. 27, 595-608.

Percus, J. \& Yevick, G. (1958). Phys. Rev. 110, 1-13.

Polizzi, S., Riello, P., Fagherazzi, G. \& Borrelli, N. (1998). J. Non-Cryst. Solids, 232/234, 147-154.

Renaud, G., Lazzari, R., Revenant, C., Barbier, A., Noblet, M., Ulrich, O., Leroy, F., Jupille, J., Borensztein, Y., Henry, C. R., Deville, J. P., Scheurer, F., Mane-Mane, J. \& Fruchart, O. (2003). Science, 300, 1416-1419.

Robach, O., Renaud, G. \& Barbier, A. (1999). Phys. Rev. B, 60, 5858-5861.

Stemmer, S., Li, Y., Foran, B., Lysaght, P. S., Streiffer, S. K., Fuoss, P. \& Seifert, S. (2003). Appl. Phys. Lett. 83, 3141-3143.

Windt, D. L. (1998). Comput. Phys. 12, 360-370. 